\title{
Transfer from perceptual pretraining as a function of number of stimulus values per dimension'
}

LOUISE S. TIGHE AND THOMAS J. TIGHE, DEPAR TMENT OF PSYCHOLOGY, DARTMOUTH COLLEGE, Hanover, $N . H$. 03755

First-grade children who were perceptually pretrained with either three stimulus values or four stimulus values appearing on each dimension in pretraining were significantly facilitated in learning a subsequent discrimination reversal as compared to both nonpretrained $S s$ and $S S$ who received identical perceptual pretraining but with only two stimulus values per dimension. These results are discussed in relation to perceptual and attentional models of learning.

Previous experiments have defined a set of pretraining conditions of a perceptual nature which produce facilitation of discrimination reversal learning in young children (Tighe, 1965; Tighe \& Tighe, 1968). The major feature of the pretraining is that Ss are required to make nonreinforced same-different judgments to successively presented stimulus objects which vary along the dimensions appearing in the subsequent discrimination task. Following the conceptions of differentiation theory (Gibson \& Gibson, 1955; Tighe \& Tighe, 1966), which provided the impetus for these experiments, it is assumed that such pretraining facilitates differentiation of the task stimuli and that positive transfer to discrimination following such treatment is due to an increase in S's sensitivity to the distinguishing features (dimensions) of the task.

An intriguing finding in one of these experiments is that facilitation of reversal obtained when four stimulus values appeared on each dimension in pretraining but not when only two stimulus values specified each dimension. This finding may be an important one for understanding the development of discrimination, especially since it has a counterpart in studies of transposition. Several investigators (e.g., Johnson \& Zara, 1960; Sherman \& Strunk, 1964) have reported that the ability of young children to transpose can be increased by "double discrimination" training in which the $S$ initially learns a discrimination involving two different pairs of stimuli lying on the same continuum. Since the number of stimulus values per dimension appears to be an important variable in both reversal and transposition behavior, it was decided to further investigate this training condition. The present experiment seeks to replicate and extend the previous finding by comparing transfer to reversal learning from perceptual pretraining with two vs three vs four stimulus values per dimension.

Method

The Ss were 64 first-grade children (average age 6 years, 7 months) randomly divided into four equal groups for differential pretraining treatment. For Ss perceptually pretrained with four stimulus values per dimension, the pretraining stimuli were 16 $1-5 / 8$ in. diameter wood cylinders which varied in height $(\mathrm{Ht})$ and brightness $(\mathrm{Br})$. Brightness was varied by covering the cylinders with black (B) or white (W) enamel paint or with one of the following enamel mixtures-dark gray ( 1 part $W$ to 4 parts $B$ ) or light gray ( 1 part B to 124 parts W). The Ht values were 4 in., 4-3/4 in., $6-1 / 4$ in., and 7 in. The 16 stimuli represented the possible combinations of these $\mathrm{Ht}$ and $\mathrm{Br}$ values. Each $\mathrm{S}$ was allowed to briefly view a standard (St) cylinder of a given $\mathrm{Ht}$ and $\mathrm{Br}$ and was asked to pretend that this object was his. He was then shown a series of successively presented comparison (Co) cylinders and required to state whether each Co stimulus was the same as his or was different. The series included the 16 possible combinations of the four $\mathrm{Ht}$ and four $\mathrm{Br}$ values, plus from one to three interspersed reappearances of the St cylinder. Two such series were given with each of the four following St stimuli: a B cylinder 4-3/4 in. high, a B cylinder 6-1/4 in. high, a W cylinder $4-3 / 4$ in. high, and a $W$ cylinder 6-1/4 in. high. Ss perceptually pretrained with two or with three stimulus values per dimension received training identical to that given Ss with four stimulus values per dimension with the exception of the stimulus objects constituting the comparison series. When only two stimulus values appeared on each dimension, E presented only $B$ or $W$ and $4-3 / 4$ in. or $6-1 / 4$ in. high cylinders, and each St was accompanied by a series of $4 \mathrm{Co}$ stimuli which represented the possible combinations of these $2 \mathrm{Ht}$ and $2 \mathrm{Br}$ values. When three stimulus values appeared on each dimension, $\mathrm{E}$ presented the $\mathrm{Ht}$ and $\mathrm{Br}$ values appearing in the two value condition plus a medium gray ( 1 part $B$ to 3 parts $W$ ) and a $5-1 / 2$ in. high cylinder. Under this condition the Co stimuli consisted of the nine possible combinations of these three $\mathrm{Ht}$ and three $\mathrm{Br}$ values. In both the two and three values per dimension conditions, as in the four value condition, the St reappeared from one to three times during any comparison series presentation. None of the judgments in any pretraining group were reinforced or corrected and all were based solely upon vision. The number of experiences with each Co and St stimulus was the same for all pretraining conditions-eight experiences per stimulus. The final pretraining treatment was a control condition in which Ss worked on tasks unrelated to the subsequent discrimination tasks and designed to control for nonspecific transfer effects. The tasks were modifications of the Picture Completion and Picture Arrangement subtests of the Wechsler Intelligence Scale for Children.

As soon as pretraining was completed all Ss learned the following two problems: (a) a 2-dimensional discrimination and (b) a reversal of that discrimination. The stimuli in these tasks were identical to the Sts used in pretraining, and were presented on a simple turntable device in the following two pairs: the tall ( $\mathrm{T}$, 6-1/4 in.) white (W) cylinder vs the short (S, 4-3/4 in.) black (B) cylinder, and the TB cylinder vs the SW cylinder. The $S$ made his choice by picking up one of the objects on each trial and, if correct, found a marble under it. The $S$ was told that the "game" was to see how soon he could find a marble every time he chose. Tall, S, B, and W were used equally often as the positive stimulus within all training conditions, and each $S$ was assigned to groups and positive stimuli on a predetermined random basis. When $S$ made 9 correct out of 10 successive responses, the discrimination reversal was presented immediately and continued to the same criterion without change of instructions. During reversal the irrelevant dimension continued to vary within trials. Throughout training the stimuli were presented according to a prearranged sequence designed to control for order and position effects. At the end of the experiment $S$ was allowed to choose a prize from an assortment which included charms, bubble gum, whistles, Hershey bars, raisins, jack sets, Tootsie rolls, pencils, pencil sharpeners, $M$ $\& \mathrm{Ms}$, and flutes.

Results

There were no differences among pretraining groups in speed of learning the initial discrimination $(\mathrm{F}<1)$. Table 1 presents the mean number of trials and errors to criterion during reversal for each group. Factorial analysis of variance applied to the trial scores transformed to $\sqrt{\mathrm{X}+.5}$ yielded a main effect of pretraining treatment $(F=4.40, \mathrm{df}=3 / 56, \mathrm{p}<.01)$. Neither the dimension assigned as relevant nor the interaction of pretraining treatment and dimension were significant sources of variance (both $F$ values

Table 1

Mean Number of Errors and Trials to Criterion during Reversal (each group contains $16 \mathrm{Ss}$ )

\begin{tabular}{lcc} 
Group & Errors & Trials \\
\hline Control & 11.3 & 20.4 \\
Two values & 10.1 & 20.6 \\
Three values & 4.8 & 8.1 \\
Four values & 5.2 & 7.3 \\
\hline
\end{tabular}


less than 1.0). By $t$ tests, the control Ss reversed significantly more slowly than both the Ss pretrained with three stimulus values per dimension $(t=2.51, p<.02)$ and the Ss pretrained with four values per dimension $(t=2.78, p<.01)$. Similarly. Ss trained with two values per dimension reversed more slowly than both $S$ trained with three values $(t=2.36, p<.05)$ and $S$ s trained with four values $(t=2.62, p<.02)$. There were no differences in speed of reversal between groups trained with three vs four values per dimension $(t=.32, p>.70)$ nor between the controls and $S s$ pretrained with two values per dimension $(t=.05, p>.90)$. All probabilities are 2-tailed. Identical conclusions result from analysis of the error measure.

Discussion

This experiment confirms the previous finding (Tighe \& Tighe, 1968) that reversal learning is facilitated when four stimulus values specify each dimension in the perceptual pretraining procedure but not when only two values are used, and it demonstrates that learning is also facilitated when three values appear on each dimension. In the previous experiment appropriate controls established that the differential effect of number of stimulus values per dimension was not due to the greater number of stimulus exposures or longer training time required by the four value as compared to the two value condition. Hence, the effect appears to be mediated directly by the variable itself. Perceptual and attentional models of discrimination learning (e.g., Sutherland, 1959: Tighe \& Tiglıe, 1966: Zeaman \& House, 1963), which relate ease of reversal learning to the degree to which discriminative response is under the control of the relevant dimension, suggest two hypotheses which might account for these data. The first is that degree of perceived continuity among stimulus values may be a critical factor in the isolation of dimensions of this type. That is, when three or four stimuli, as opposed to two stimuli, specified each dimension, the children may have been more apt to perceive their relative properties and therefore to respond to them as a distinct class of cues. The second hypothesis is that the smaller distance between stimulus values in the three and four value condition evoked greater perceptual search and thus made more likely the isolation of the task dimensions. These hypotheses are readily tested by comparing the present two value pretraining condition with (a) a condition in which Ss are allowed to experience continuous variation between these two values and (b) a condition in which Ss experience two values but separated by a smaller distance. Finally, this experiment has implications for the previously cited findings of a facilitating effect of "double discrimination" training on transposition. This observation has been interpreted as a learning set mechanism, but the present results, particularly the finding that facilitation obtained with three values as well as with four values per dimension, indicate that a perceptual learning process of the type suggested here is involved.

\section{REFERENCES}

GIBSON. J. J., \& GIBSON, E. J. Perceptual learning: differentiation or enrichment? Psychol. Rev., 1955, 62, 32-41.

JOHNSON, R. C., \& ZARA, R. C. Relational learning in young children. $J$. comp. physiol. Psychol., 1960, 53, 594-597.

SHERMAN, M., \& STRUNK, J. Transposition as a function of single versus double discrimination training. J. comp. physiol. Psychol., 1964, 58, $449-450$.

SUTHERLAND, N. S. Stimulus analyzing mechanisms. In, Proceedings of a symposium on the mechanization of thought processes. Vol. 2. London: Her Majesty's Stationery Office, 1959. Pp. 575-609.

TIGHE, L. S. Effect of perceptual pretraining on reversal and nonreversal shifts. J. exp. Psychol., 1965, 70, 379-385.

TIGHE, L. S., \& TIGHE, T. J. Discrimination learning: Two views in historical perspective. Psychol. Bull., 1966, 66, 353-370.

TIGHE, T. J., \& TIGHE, L. S. Perceptual learning in the discrimination processes of children: An analysis of five variables in perceptual pretraining. J. exp. Psychol., 1968, 77, 125-134.

ZEAMAN, D., \& HOUSE, B. J. The role of attention in retardate discrimination learning. In N. R. Ellis (Ed.), Handbook of mental deficiency. New York: McGraw-Hill, 1963. Pp. 159-223.

\section{NOTE}

1. This research was supported by Public Health Service Research Grant MH1 1088, from the National Institute of Mental Health. The authors are grateful for the generous cooperation of Gordon Tate, Superintendant of the Lebanon. N. H. school system and for the assistance of the principals and teachers of this school system. We also wish to acknowledge the contribution of Susan Greene who assisted in collecting the data. 